\title{
Spiral Wave Law Is Involved in the Unification of Four Fundamental Forces and the Origin and the Evolution of the Universe
}

\section{Jiapei Dai}

Wuhan Institute for Neuroscience and Neuroengineering, South-Central University for Nationalities, Wuhan, China

Correspondence to: Jiapei Dai, Jdai@mail.scuec.edu.cn

Keywords: Spiral Wave Law, Fundamental Forces, Origin and the Evolution of the Universe

Received: August 8, $2017 \quad$ Accepted: September 16, 2017

Published: September 19, 2017

Copyright @ 2017 by authors and Scientific Research Publishing Inc.

This work is licensed under the Creative Commons Attribution International License (CC BY 4.0).

http://creativecommons.org/licenses/by/4.0/

(c) (i) Open Access

\section{ABSTRACT}

A long-term goal of theoretical physics is to develop a single simple theory or model that would unify the four known fundamental forces (or interactions) and give explanations for the origin and the evolution of the Universe. Here a "spiral wave law" has been proposed based on the previous studies that a consistent universe field presents various forms of spiral (helical) wave motions at the speed of light (c), and therefore, a mathematical equation for the relationship between the radius of a spiral wave motion $(r)$ and its wave length $(\lambda)$ is derived including a simplified formula $\left(\lambda=2 \pi r^{2}\right.$ or $\left.r=\sqrt{c t / 2 \pi}\right)$, which could provide a novel explanation for the origin and the evolution of the Universe, and the space-time relationships. This model may give a new way for the unification of four fundamental forces and determine the moving properties of galaxies and basic particles, and the propagation characteristics of electromagnetic waves at the large or small scale.

\section{INTRODUCTION}

A long-term goal of theoretical physics is to develop a single simple theory or model $[1,2]$ that would unify the four known fundamental forces (or interactions) and give explanations for the origin and the evolution of the Universe, and other physical questions or problems, such as the origin of the gravity and mass [3], the nature of dark matter and dark energy [4, 5], the horizon problem [6], the flatness/oldness problem [7], the strong CP problem [8], the neutrino oscillations [9, 10], and the baryon asymmetry [11-13], etc. However, such a theory still does not appear.

\section{THE DERIVATION OF SPIRAL WAVE LAW}

It can be assumed that "Ether" or "Aether" that has been debated for more than five centuries in physics is existed based on the increasing research evidence [14], called the "universe field", which was 
proposed before [15], and here I want to derive a spiral wave law according to this report. There have three distinct characters for the proposed universe field: 1) The universe field presents a spiral (helical) wavelike motion at the speed of light at the small scale [15] (Figure 1);2) A microscopic matter is just the particular energy form of a spiral wavelike motion companying with a spin movement in the universe field, named as "spin wave packet", which forms macroscopic objects; and 3) There exist the transformation between the universe field energy $\left(E_{u}\right)$ and the matter energy $\left(E_{m}\right)$ in the Universe, which is mediated via electromagnetic waves.

We cannot directly assess $E_{\iota}$, however, we could define it when considering of energy transformation from $E_{m}$ to the part of $E_{u}\left(p E_{u}\right)$. Therefore, according to the energy conservation law and the mass energy formula, we can write an equation as follows:

$$
p E_{u}=E_{m}=m c^{2}
$$

where " $c$ " is the speed of light and " $m$ " is the quality of a matter. It may be more understandable to evaluate the $E_{m}$ by taking a photon as an example rather than a macroscopic object. If the quality of a photon is " $m$ " (actually, $m$ is considered as a wave packet in order to avoid arguments on whether a photon has the quality or not) and its whole momentum is $P_{W}$ that includes the spiral (helical) angular momentum $\left(P_{h}\right)$ and the spin momentum $\left(P_{s}\right)$ (Figure 2), then

$$
P_{w}=P_{h}+P_{s}
$$

Because a photon presents a spiral wave motion at the small scale according to the assumption mentioned above, and therefore, if the linear velocity of spiral (helical) wave movement of a photon is " $v$ "; then

$$
v=s / t
$$

where " $s$ " is a spiral circumference, and " $t$ " is the time for a spiral wave motion of a photon (Figure 2); then

$$
v=2 \pi r / t
$$

where " $r$ " is the radius of helix

The time for a spiral wave motion of a photon can also be determined by

$$
t=\lambda / c
$$

where " $\lambda$ " is the wavelength of spiral wave motion; then

$$
v=2 \pi c r / \lambda
$$

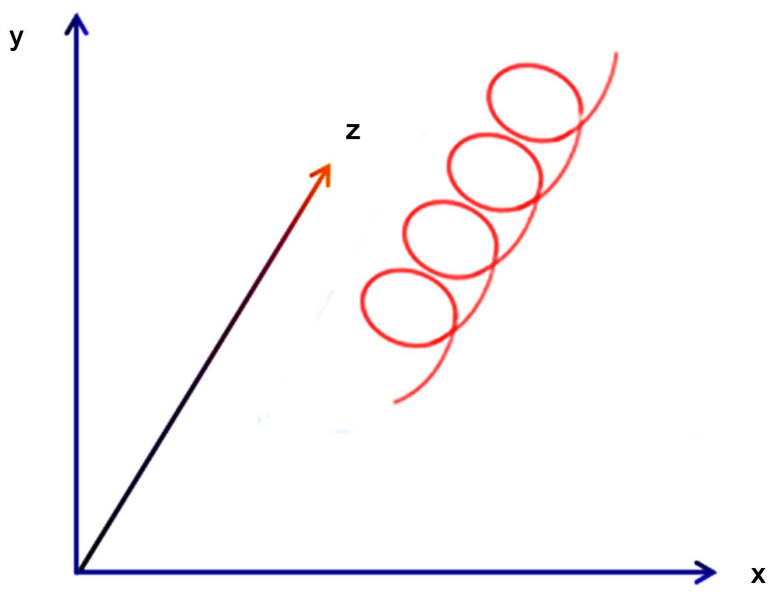

Figure 1. Schematic drawing of the spiral (helical) wave motion of the universe field at the small scale in a four-dimensional space ( $x, y, z$ and $t)$. 


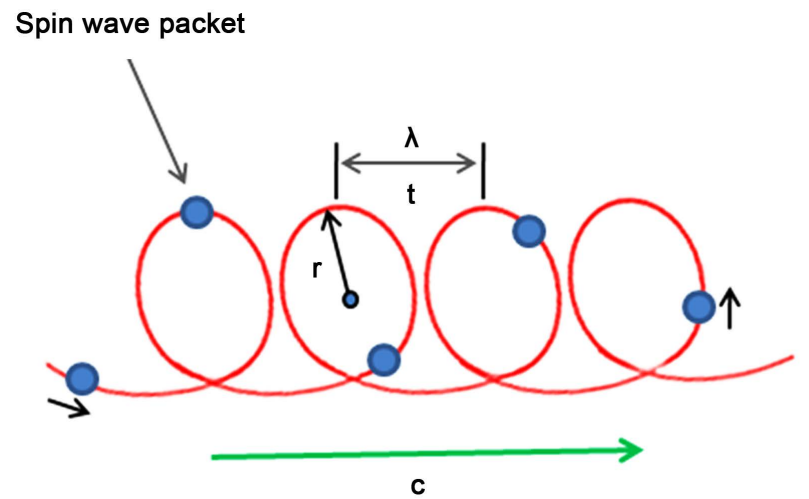

Figure 2. Schematic drawing of the spiral (helical) wave motion of a photon in the universe field for deriving a unifying mathematical equation. $c, r, \lambda$ and $t$ is the speed of light, the radius of helix, the wavelength of spiral wave motion and the time for a spiral wave motion, respectively. The blue circular spots show the wave packets of a matter such as a photon, presenting a spiral wave motion companying with the spin movement (or spin momentum).

According to the angular momentum law, then

$$
P_{h}=r(m v)=2 \pi r^{2} m c / \lambda
$$

Because $P_{w}=E_{m} / c=m c^{2} / c=m c$ for a photon, then

$$
\begin{aligned}
& 2 \pi r^{2} m c / \lambda+P_{s}=m c \\
& \lambda=2 \pi r^{2} m c /\left(m c-P_{s}\right)
\end{aligned}
$$

\section{DISCUSSION}

This is a unifying equation for the movement of a matter and its relationship to the universe field, and is defined as a "spiral wave law" (SWL). Such a mathematical equation can be used to describe the movement rules of the universe field and the matters such as the galaxies and planets, the basic particles and the electromagnetic waves both at the macroscopic and microscopic levels. In addition, the wave-particle duality of mater is unified in this mathematical equation.

For example, if $P_{s}=0$, meaning that the spin movement or momentum of a particle such as a photon is disappeared, and consequently the particle is completely transferred into the universe energy (the universe field), then

$$
\lambda=2 \pi r^{2}
$$

or

$$
\lambda / r^{2}=2 \pi=\text { constant }
$$

or if $\lambda=c t$, then

$$
r=\sqrt{c t / 2 \pi}
$$

These are three mathematical equations for the wave features of the university field, and the cosmic physical connotations contain: 1) The appearance of the Universe presents a shuttle shaped sphere; 2) If " $r$ " $\rightarrow 0$, then " $\lambda$ " $\rightarrow 0$, indicating that a singularity occurs, which may be the origin of the Universe; 3 ) If " $c$ " is variable, for example " $c$ " $\rightarrow 0$, then " $r$ " $\rightarrow 0$, indicating that a black hole may be appeared under the condition of universe field collapse, in which the formed matter may just have spin movement or spin 
momentum, and the spiral wave movement of the universe field is disappeared there. Because the " $c$ " value is limited (for example, $c \neq \infty$ ), then " $r$ " is also limited, indicating that the Universe is bounded; and 4) The Equation (10c) describes the space-time relationship (or law). If " $t$ " is increasing, then the space is expanding, but it could not do so forever, indicating that the accelerated expansion of the Universe may just be a local process of the cyclic motion of the Universe [16] because there exist three possibilities including the accelerating expansion (red shift), accelerating shrink (blue shift) and unaltered condition (invariant spectral feature) of the Universe based on the observation of the spectral information between the stars (Figure 3).

The macroscopic objects such as the sun and earth, etc, also present a spiral wavelike motion, and their movement characteristics including the wavelength and radius of spiral wave like motion can be determined by the mathematical Equation (9). We take the sun's planets as examples:

The spin angular momentum of a planet can be described by as implified equation as follows:

$$
P_{s}=I \omega
$$

where " $P$ " and " $\omega$ " are the moment of inertia and the average spin angular velocity of a planet, respectively, then

$$
\lambda=2 \pi r^{2} m c /(m c-I \omega)
$$

Therefore, if the average radius of the revolution of a planet is considered as its radius of the spiral wave movement, then the $\lambda$ of the sun's planets can be calculated according to the other measured parameters $(m, I$ and $\omega)$ of these planets.

It could be proposed that the wavelengths of spiral wavelike motion of the sun's planets are identical to their wavelengths of gravitational waves, indicating that the gravity originates from the gravitational waves. In addition, the trajectory of spiral wavelike motion of the sun's planets is curved, which may cause the precession of the sun's planets.

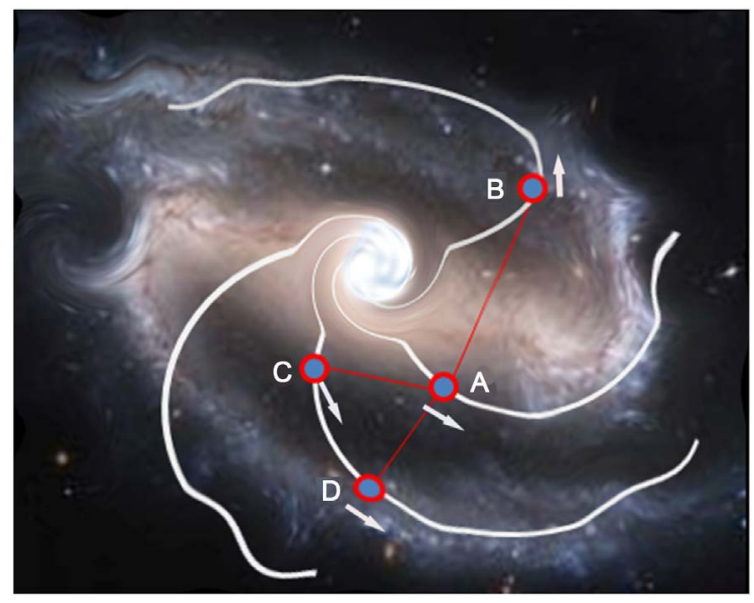

Figure 3. Schematic drawing of the three possibilities of movement relationships between the stars in the Universe. Arrows indicate the movement directions of four stars (A-D). If the star B moves away the star A, then observations in $A$ would result in redshirt effects; if the star $C$ moves close to the star A, then would result in blue shift effects, and if the star D and $A$ present motion synchronization, then would result in invariant spectral features. Such relationships indicate that the accelerating expansion, accelerating shrink and unaltered condition of the Universe may be existed based on the observations of spectral information. 
The microscopic particles and the electromagnetic waves also present spiral wave like motions, and according to the quantum mechanics [17], the spin angular momentum of a particle can be determined by an equation as follows:

$$
P_{s}=h \sqrt{j(j+1)}
$$

where " $j$ " is the spin quantum number, $j=0, \frac{1}{2}, 1, \frac{3}{2}$, etc., and " $h$ " is the Planck constant, then

$$
\lambda=2 \pi r^{2} m c /[m c-h \sqrt{j(j+1)}]
$$

This is a mathematical equation for the spiral wave movement of particles.

In this study, a unified theory has been proposed, which would unify the four known fundamental forces (or interactions) and give explanations for the origin and the evolution of the Universe. Therefore, it could be concluded that the nature of the universal gravitation is due to the interaction between the matters via the gravitational waves at the large scale, and similarly, the electromagnetic force, the strong and weak interactions hold same mechanism as gravity at the small scale. In addition, this unified theory may provide novel explanations for other physical questions or problems, such as the origin of the gravity and mass, the nature of dark matter and dark energy, the horizon problem, the flatness/oldness problem and the neutrino oscillations.

It should be particularly emphasized that the spiral wavelike motion of the sun (the origin of sun's gravitational wave) and its action on the earth may be the causes of nature occurred climate phenomena on earth such as typhoons and hurricanes, etc., which usually present spiral wavelike motions and are related to the specific seasonal periods when the sun's gravitational wave may generate the maximum effects on the earth. In addition, the lens effect of the propagation of light in the Universe is involved in the gravitational waves of the stars such as the sun.

\section{ACKNOWLEDGEMENTS}

This work was supported by the research team fund of South-Central University for Nationalities (XTZ15014).

\section{COMPETING FINANCIAL INTERESTS}

The authors declare no competing financial interests.

\section{REFERENCES}

1. 'tHooft, G., et al. (2005) A Theory of Everything? Nature, 433, 257.

2. Waldrop, M.M. (2011) Unification + 150. Nature, 471, 286. https://doi.org/10.1038/471286a

3. Abbott, B.P., et al. (2016) GW150914: The Advanced LIGO Detectors in the Era of First Discoveries. Physical Review Letters, 116, Article ID: 061102. https://doi.org/10.1103/PhysRevLett.116.131103

4. Peebles, P.J. (2015) Dark Matter. Proceedings of the National Academy of Sciences USA, 112, Article ID: 12246. https://doi.org/10.1073/pnas.1308786111

5. Wang, B., Abdalla, E., Atrio-Barandela, F. and Pavón, D. (2016) Dark Matter and Dark Energy Interactions: Theoretical Challenges, Cosmological Implications and Observational Signatures. Reports on Progress in Physics, 79, Article ID: 096901. https://doi.org/10.1088/0034-4885/79/9/096901

6. Chung, D.J.H. and Freese, K. (2000) Cosmological Challenges in Theories with Extra Dimensions and Remarks on the Horizon Problem. Physical Review D, 61, 1.

7. Lightman, A. (1993) Ancient Light: Our Changing View of the Universe. Harvard University Press, Cambridge. 
8. Mainini, R. and Bonometto, S.A. (2004) Dark Matter and Dark Energy from the Solution of the Strong CP Problem. Physical Review Letters, 93, Article ID: 121301. https://doi.org/10.1103/PhysRevLett.93.121301

9. Hasert, F.J., et al. (1973) Observation of Neutrino-Like Interactions without Muon or Electron in the Gargamelle Neutrino Experiment. Physics Letters B, 46, 138. https://doi.org/10.1016/0370-2693(73)90499-1

10. Vogel, P., Wen, L.J. and Zhang, C. (2015) Neutrino Oscillation Studies with Reactors. Nature Communications, 6, Article ID: 6935. https://doi.org/10.1038/ncomms7935

11. Glashow, S.L. (1961) Partial-Symmetries of Weak Interactions. Nuclear Physics, 22, 579. https://doi.org/10.1016/0029-5582(61)90469-2

12. Farrar, G.R. and Zaharijas, G. (2006) Dark Matter and the Baryon Asymmetry of the Universe. Physical Review Letters, 96, Article ID: 041302. https://doi.org/10.1103/PhysRevLett.96.041302

13. Cho, A. (2002) Particle Physics. Hints of Greater Matter-Antimatter Asymmetry Challenge Theorists. Science, 328, 1087. https://doi.org/10.1126/science.328.5982.1087-a

14. Ranzan, C. (2016) The History of the Aether Theory. A Compendious Summary and Chronology of the Aether Theories. Cellular Universe Website.

15. Dai, J. (2012) Universe Collapse Model and Its Roles in the Unification of Four Fundamental Forces and the Origin and the Evolution of the Universe. Natural Science, 4, 199. https://doi.org/10.4236/ns.2012.44030

16. Steinhardt, P.J. and Turok, N. (2002) A Cyclic Model of the Universe. Science, 296, 1436. https://doi.org/10.1126/science.1070462

17. Griffiths, J.D. (2005) Introduction to Quantum Mechanics. 2nd Edition, Pearson Education Limited, London, 183-184.

\section{Submit or recommend next manuscript to SCIRP and we will provide best service for you:}

Accepting pre-submission inquiries through Email, Facebook, LinkedIn, Twitter, etc.

A wide selection of journals (inclusive of 9 subjects, more than 200 journals)

Providing 24-hour high-quality service

User-friendly online submission system

Fair and swift peer-review system

Efficient typesetting and proofreading procedure

Display of the result of downloads and visits, as well as the number of cited articles

Maximum dissemination of your research work

Submit your manuscript at: http://papersubmission.scirp.org/

Or contact ns@scirp.org 